\title{
Potential impact of antiarrhythmic drugs versus implantable defibrillators on the management of ventricular arrhythmias: the Midlands trial of empirical amiodarone versus electrophysiologically guided intervention and cardioverter implant registry data
}

\author{
R K Pathmanathan, E W Lau, J Cooper, L Newton, J D Skehan, C J Garratt, M J Griffith
}

\begin{abstract}
Background-Survival was prolonged in selected patients with sustained ventricular arrhythmias who received implantable cardioverter defibrillators (ICDs) in the antiarrhythmics versus implantable defibrillators (AVID) study. The Midlands trial of empirical amiodarone versus electrophysiologically guided intervention and cardioverter implant in ventricular arrhythmias (MAVERIC) registry is a
\end{abstract} population based trial.

Objective-To determine the number of patients who satisfy the AVID criteria because of the high cost of ICDs.

Design-Observational study, based on a continuing trial.

Setting-All coronary care units in the Midlands region in the United Kingdom (population 9.1 million).

Patients-Patients presenting to a coronary care unit with sustained ventricular arrhythmias not related to an acute myocardial infarction are entered onto the registry. Those who consent to the MAVERIC study are randomised to receive either empirical amiodarone or electrophysiologically guided treatment. Demographic data, details of clinical presentation, and echocardiographic findings are collected. These data have been used to calculate the number of patients who satisfy the AVID criteria and would benefit from ICD implantation. The financial implications have been calculated for the region and nationally.

Results-132 patients were entered onto the registry during the first five months of the MAVERIC study; 69 patients fulfilled the AVID criteria. Extrapolation of these data over a 12 month period suggests implantation of at least 166 new ICDs (compared with 23 implants in 1996). This would increase the UK ICD implant rate from five to at least 18 per million of the population, costing the National Health Service $£ 24.1$ million per annum.

Conclusion-Application of the AVID criteria in the UK will cause a great increase in the ICD implant rate, with serious financial implications.

(Heart 1998;80:68-70)
Keywords: AVID study; MAVERIC trial; implantable cardioverter defibrillator; economics; arrhythmias

Sustained ventricular tachyarrhythmias not related to an acute myocardial infarction are a major cause of sudden cardiac death in the UK. Survivors of cardiac arrest are at high risk of further arrests. ${ }^{1}$ Initial treatment is often empirical amiodarone. Referral for specialist electrophysiological assessment usually occurs only if the patient is fortunate to survive a further episode of sustained ventricular arrhythmia. Implantable cardioverter defibrillators (ICDs) are effective at preventing sudden cardiac death in patients with recurrent life threatening ventricular arrhythmias, and by implication of prolonging life in these patients. ${ }^{23}$ As ICD implantation is very expensive and many patients who may benefit have a poor prognosis because of severely impaired ventricular function, a prospective randomised trial is needed to demonstrate their efficacy.

The antiarrhythmics versus implantable defibrillators (AVID) study, ${ }^{4}$ in which patients with haemodynamically poorly tolerated ventricular arrhythmias were randomised to receive either ICD implantation or antiarrhythmic treatment, was terminated prematurely because of significantly improved mortality in patients receiving ICDs (results presented at the 18 th annual scientific NASPE meeting in New Orleans in May 1997). This is the first randomised study to show a clear benefit of ICD implantation for selected patients with sustained ventricular arrhythmias.

We have calculated the number of patients in the UK who would fulfil the AVID criteria and therefore benefit from an ICD, using information from our ongoing prospective population based randomised study of the management of ventricular arrhythmia (MAVERIC).

\section{Methods}

THE MAVERIC STUDY

MAVERIC, a prospective, population based study in the Midlands of the UK, is coordinated from Birmingham and Leicester. Patients with sustained ventricular arrhythmias are randomised to receive either empirical amiodarone or electrophysiologically guided intervention, which can be one or a combina- 
tion of antiarrhythmic drugs, coronary revascularisation, or ICD implantation. Active recruitment started on 1 February 1997. We have set a target to recruit 200 patients in two years.

The inclusion criteria are resuscitated ventricular fibrillation or tachycardia, sustained non-syncopal ventricular tachycardia, and resuscitated sudden cardiac death. The exclusion criteria are a myocardial infarction within the previous 48 hours, a prognosis of less than six months from a non-arrhythmic cause, and pregnancy.

Randomisation takes place at the presenting hospital and patients who are not randomised are entered onto a registry. Part of the aim of this study is to determine the incidence and natural history of ventricular arrhythmias unrelated to an acute myocardial infarction, in a defined geographical region with a population of 9.1 million. Thus every patient presenting to a coronary care unit with a sustained ventricular arrhythmia unrelated to an acute myocardial infarction is entered onto a registry and registered with the Office of National Statistics for follow up mortality data. Study patients are followed by regular outpatient visits, patient diaries, and quality of life questionnaires. The primary end point is death. The secondary end points are recurrence of ventricular arrhythmia, resuscitated sudden cardiac death, crossover to non-pharmacological treatment because of recurrence or intolerance of side effects, and referral of empirically treated patients for electrophysiology. The cost of the two arms of the study will also be assessed using patient diaries and careful documentation of all investigations.

\section{PATIENT RECRUITMENT}

Patients are being recruited from 36 centres, which include all coronary care units in the Midlands region. We visited each centre three to four times during the first five months of the study to increase the profile and explain the study, as well as to help recruit patients. The study has been widely publicised by letters and newsletters to physicians and cardiologists, during medical meetings at participating centres, regular contact with coronary care nursing and medical staff by weekly telephone calls, and MAVERIC posters. Each centre is visited every three months and the coronary care admissions book is carefully scrutinised to ensure that patients eligible for entry onto the registry are not missed.

Patients presenting with sustained ventricular arrhythmia without myocardial infarction within the previous 48 hours are entered onto the registry. Their demographic details, presenting arrhythmia, conscious state, and echocardiographic data are recorded.

\section{Results}

A total of 132 patients have been entered onto the registry in the first five months of the MAVERIC study. Half of these patients agreed to participate and were randomised to receive either treatment strategy. Reasons for nonentry into the study usually included the physi-
Table 1 AVID inclusion criteria

Ventricular fibrillation

Ventricular tachycardia with syncope

Ventricular tachycardia without syncope,

ejection fraction $<0.40$, and systolic $\mathrm{BP}<80 \mathrm{~mm} \mathrm{Hg}$

Table 2 Characteristics of MAVERIC registry patients fitting AVID criteria

\begin{tabular}{ll}
\hline Mean age & 65.8 years \\
Male & $46(66.7 \%)$ \\
VF or resuscitated VT & $44(63.8 \%)$ \\
Syncopal VT & $10(14.5 \%)$ \\
$\begin{array}{l}\text { Non-syncopal VT, EF }<0.40, \\
\text { systolic BP }<80 \mathrm{~mm} \mathrm{Hg}\end{array}$ & $15(21.7 \%)$ \\
\hline
\end{tabular}

Table 3 Minimum ICD implant costs

\begin{tabular}{lr}
\hline Device and lead & \\
4 days inpatient stay & $£ 18500$ \\
2 hours catheter laboratory time & $£ 800$ \\
Total & $£ 20700$ \\
Total including $9 \%$ capital costs & $£ 22563$ \\
\hline
\end{tabular}

cian's preference for a particular treatment and occasionally patient refusal.

Inclusion criteria for the AVID study (table 1) were applied to the total registry population to assess their effect. Sixty nine patients $(67 \%$ men) with a mean age of 65.8 years satisfied the AVID criteria. Almost two thirds presented with ventricular fibrillation or resuscitated ventricular tachycardia (table 2). Extrapolation of these data over a 12 month period suggests implantation of at least 166 ICDs per annum at first presentation. There were 23 new implants in the Midlands in 1996, four of which were in patients who did not satisfy the AVID criteria.

Cost is a significant factor. We calculated the lowest costs of an ICD implant based on current charges in the region. Costs for other investigations or treatments, such as coronary angiography or revascularisation, which are often necessary in such patients, were not considered. The cheapest ICDs cost approximately $£ 18500$ including value added tax. At the very least such patients would be in hospital for four days at an average cost of $£ 350 /$ day. Allowing for an average of two hours of cardiac catheter laboratory time for an implant at $£ 400$ /hour, laboratory time would cost $£ 800$. This would give a basic cost of $£ 20700$ for each ICD implant, which would increase to $£ 22563$ once capital charges of $9 \%$ are added (table 3).

On the basis of the MAVERIC registry data, at least 166 patients each year in the Midlands with an estimated total population of 9.1 million would satisfy the AVID criteria and potentially benefit from an ICD implant. This gives a mean of 18.2 new ICD implants per million population. The basic cost calculated on the assumption that each implant costs $£ 22563$ is $£ 3745458$ per annum. The minimum annual cost to the National Health Service if these values are extrapolated to the UK population of 58605782 million would be $£ 24.1$ million. If the 132 patients on the MAVERIC registry received an ICD implant, the annual implant rate per million of population would be 34.8 . 


\section{Discussion}

The first five months of the MAVERIC registry have provided an estimate of the likely minimum incidence of life threatening ventricular arrhythmias in a defined population. It also suggests that just over half of these patients would satisfy the AVID criteria. In the AVID study the average age of patients was 65 years. MAVERIC patients fulfilling the AVID criteria had a similar mean age of 65.8 years. The AVID trial was stopped prematurely in April 1997 after recruitment of 1016 patients. One year mortality was reduced by $38 \%$ in patients treated with an ICD compared with those who received antiarrhythmic drug treatment. This significantly improved prognosis continued in the second and third years, with a $25 \%$ reduction in deaths in each year.

An improvement in survival with ICD treatment has been suggested in other studies. Patients with previous myocardial infarction with non-sustained ventricular tachycardia, impaired left ventricular function, and nonsuppressible inducible ventricular arrhythmias were randomised in the multicentre automatic defibrillator implantation trial (MADIT) to receive either an ICD or conventional medical treatment. ${ }^{5}$ The ICD group had an improved survival rate. Patients at high risk of sudden cardiac death in the coronary artery bypass surgery patch study ${ }^{6}$ were randomly allocated to receive defibrillators at the time of surgical coronary revascularisation. There was no significant survival difference in the two groups despite $50 \%$ of patients with ICDs having discharges from their devices (CABG patch trial results presented at the NASPE conference in May 1997). There are also ongoing randomised defibrillator trials, such as the cardiac arrest study, Hamburg ${ }^{7}$ and the Canadian implantable defibrillator study. ${ }^{8}$

There seems to be increasing evidence that cardiologists should be more aggressive in their management of patients with life threatening ventricular arrhythmias. The MAVERIC registry data confirm what has long been suspected, that many more patients would benefit from an ICD implant. Several reasons may explain this finding but a combination of lack of sufficient evidence and the very high cost of ICDs play a significant part. Interestingly, even if the 132 patients with sustained ventricular tachyarrhythmias had ICDs fitted, the rate per million would be 34.8 . This would be an almost seven- fold increase in the current UK implant rate of 5 per million, but closer to the German and Austrian rate of 25 per million. Yet this is still only half the US implant rate of 89 per million (Medtronic estimates for all ICD manufacturers, 1996), which suggests that their selection criteria are not limited to the AVID group but must include a significant number of patients with well tolerated ventricular tachycardias or patients with supposed prophylactic indications for ICDs.

Despite our good intentions we are inevitably limited by funding constraints within the National Health Service. The sum of $£ 24$.1 million per annum spent on a relatively small group of patients who often have a reduced prognosis from left ventricular dysfunction may be considerably more than we can afford. This value is only a bare minimum: it does not consider patients who may have ICDs fitted for other reasons such as those satisfying the MADIT criteria. In the Midlands in 1996, 19 of 23 new implants (83\%) fulfilled the AVID criteria. This means that an additional $17 \%$ of patients may have an ICD implant on the basis of other criteria, thus increasing further the ICD cost burden. The cost of renewal units and follow up care will add further to the overall cost of ICD treatment. For these reasons, it is important that the longer term cost effectiveness rather than just the medium term survival benefit of these devices in the UK setting is determined in a prospective study.

1 Cobb LA, Baum RS, Alvarez H, et al. Resuscitation from out-of-hospital ventricular fibrillation: 4 year follow-up. Circulation 1975;51:III-223-8.

2 Mirwoski $M$. The automatic implantable cardioverterdefibrillator: an overview. $\mathcal{F}$ Am Coll Cardiol 1985;6:461-6.

3 Newman D, Sauve MJ, Herre J, et al. Survival after implantation of the cardioverter defibrillator. $\mathrm{Am} f$ Cardiol 1992;69:899-903.

4 The AVID investigators. Antiarrhythmics versus implantable defibrillators (AVID) - rationale, design, and methods. Am 7 Cardiol 1995;75:470-5.

5 Moss AJ, Hall WJ, Cannom DS, et al for the Multicenter Automatic Defibrillator Implantation Trial Investigators Automatic Defibrillator Implantation Trial Investigators (MADIT). Improved survival with an implanted defibrilla(Misk for Spotnitz HM, Herre JM, Baker LD, et al. Surgical aspects of a randomised trial of defibrillator implantation during coronary artery bypass surgery-the CABG patch trial. Circulation 1996;94(9):II-248-53.

7 Siebels J, Kuck KH. Implantable cardioverter defibrillator compared with antiarrhythmic drug treatment in cardiac arrest survivors (the cardiac arrest study Hamburg). $\mathrm{Am}$ Heart f 1994;127:1139-44.

8 Connolly SJ, Gent M, Roberts RS, et al. Canadian implantation defibrillator study (CIDS): study design and organization. Am 7 Cardiol 1993;72:103F-8F. 